\title{
Optimizing routines for heat treatment of metals using numerical simulation based on infrared thermographic measurement
}

\author{
by I. Sundov
}

University of Zagreb, Faculty of Mechanical Engineering and Naval Architecture, I. Lučića 5, Zagreb, Croatia, E-mail: isundov@fsb.hr

\begin{abstract}
:
The analysis of complex probe geometry heating and cooling was done aiming to find the temperature distribution inside the probe versus time. An infrared thermography was used to measure surface temperatures while temperatures inside the probe were measured with thermocouples. The results obtained by means of the numerical model based on the control volume approach, where thermographic measurements were the input parameters, show good mutual accordance with the ones obtained by thermocouple measurement at referent points of the probe. The local and average heat transfer coefficients have also been calculated by means of a numerical model. Based on that data, it is possible to find the influence of heat transfer level on heating or cooling processes. The simulation of applied therodynamics problems, especially in heat treatment of metals, provides a possibility to predict and monitor temperature change for each single point inside the probe in a way which will guarantee that the desired microstructure is obtained. The goal of the project was to find a possibility to simulate similar processes without providing measurements for all possible setups of the furnace parameters, which will reduces costs and time.
\end{abstract}

\section{Introduction}

Heat treatment of metals is a set of techniques with the aim to change properties of the material. The reached microstructure is caused by chemical structure (Carbon share especially) and by changing to more or less fine crystal grate, which is the result of faster or slower changes of temperatures of each single point inside a metal body. Depending on metal part purpose, an adequate heat treatment technique should be chosen (tempering, quenching, annealing). Figure 1 shows an example in CTT (Continuos Cooling Transformation) diagram for quenching for one type of steel with temperature cooling curves for three different points inside the metal body. Abscissa axis shows the final reached hardness and it is obvious that hardness rises as temperature cooling curve is closer to the ordinate axis.

No matter which technique should be applied, neither of metal heat treatment techniques will be used alone (almost never). It is usual that quenching is preceded by annealing and followed by at least one tempering cycle. Figure 2 shows an example of a complete heat treatment procedure. It is obvious that each heat treatment technique consists of heating followed by cooling. Thus it is important to know the ending temperature distribution inside metal part after every heating or cooling procedure.

It is possibele to calculate the temperature distribution inside metal body versus time during heating and cooling by solving differential equation for non-steady heat conduction.

$\frac{\partial}{\partial x}\left[\lambda(x, y, z, t) \frac{\partial T}{\partial x}\right]+\frac{\partial}{\partial y}\left[\lambda(x, y, z, t) \frac{\partial T}{\partial y}\right]+\frac{\partial}{\partial z}\left[\lambda(x, y, z, t) \frac{\partial T}{\partial z}\right]+\Phi_{v}=\rho c(x, y, z, t) \frac{\partial T}{\partial t}$

Analytical solution of equation is possible for one-dimensional treated problems but for simple geometry only (cylinder, plate). It should be pointed out that non-uniformity of the start temperature distribution could not be treated with analytical approach. When there is no other solution, numerical approach is the last to be applied.

\section{The problem}


It has already been pointed out that for metal heat treatment techniques is important to know the temperature distribution inside the probe versus time. This data provide basic information whether the process goes on in the proper way or not, and these data are directly related to furnace parameters. The metal heat treatment techniques are mostly rely on experimental or empirical data. There are some methods for calculating heating and cooling time, but most of them have been developed for metal parts with the simple geometry. The main problem for those methods is unsatisfactory precision. If there is any control measurement during the application of some of the techniques, it is realised by means of a specially designed probe equipped with few thermocouples inside the probe.

The contactless measurement of the surface temperature supported with adequate software for the calculation of the temperature distribution inside the specimen will be a better quality solution, and the basis for the development of the heat treatment monitoring system. The goal of the project was to develop a numerical model where the infrared measurements will be the input parameters, which will ensure a good accuracy of results with data obtained by thermocouples, positioned in control points inside the probe. The possibility to predict and monitor temperature change for each single point inside the probe allows controlling the heat treatment system in a way which will guarantee that the desired microstructure is obtained (cooling curves in CCT diagram).

\section{Experimental set-up}

The analysis of the probe with complex geometry (fig. 3) exposed to heating and cooling was done with the aim to find the temperature distribution inside the probe versus time. The probe is made of steel W. Nr. 1.4301 (DIN) with the following chemical composition: $\mathrm{C}=0,031 \%, \mathrm{Si}=$ $0,57 \%, \quad M n=1,4 \%, \quad P=0,028 \%, \quad S=0,007 \%, \quad C r=18,48 \%, \quad \mathrm{Ni}=9,08 \%$. Physical characteristics of steel in temperature range between $0^{\circ} \mathrm{C}$ and $1000{ }^{\circ} \mathrm{C}$ are defined with the following equations:

$$
\begin{aligned}
& \lambda=0,013564 \vartheta+15,127 \\
& c=1,3410^{-7} \vartheta^{3}-2,9110^{-4} \vartheta^{2}+0,28 \vartheta+450 \\
& \rho_{\vartheta}=\rho_{20^{\circ} C}-0,45 \vartheta
\end{aligned}
$$

The surface temperatures were measured by infrared camera (fig. 4), and the temperatures inside the probe by thermocouples. Thermocouples 1, 5 and 6 (fig. 3) were used to measure surface temperature, while thermocouples 2, 3, 4 and 7 were used to control numerical solution. The experiments were carried out on the experimental rigs for heating (fig. 5) and cooling (fig. 6) specially designed for that purpose. Thermographic measurements were carried out by two infrared systems: AGA 680 standard and AGEMA 470 PRO. The probe was painted with colour with known emissivity factor, $\varepsilon=0,97$.

The heating experiments were carried out in furnace with reconstructed small door (open during heating). Walls in the furnace were covered with thick steel plates equipped with thermocouples. The probe was inserted into cold furnace, and heated to the desired temperature. Together with thermocouples positioned on the probe 17 temperatures were measured with $A D$ converter, connected to a PC during the whole period of heating, while surface temperature distribution was taken in steps by means of thermographic system. The probe was, after being heated to a desired temperature, inserted into the air stream vertically, and the measuring procedure was quite similar to heating one. Cooling experiments were examined in the air streams at the velocities of 1,5 m/s, 2 $\mathrm{m} / \mathrm{s}, 3 \mathrm{~m} / \mathrm{s}$, and in the still air. The results of thermographic measurements were used as input data for mathematical model based on the control volume method. 


\section{Simulation}

The numerical model for determining the temperature distribution inside the probe was based on the differential equation of unsteady heat conduction expressed in polar coordinates (5). The temperature distribution was analyzed as two-dimensional problem, differential equation was discretized (6) and the system of algebraic equations solved by ADI algorithm.

$$
\begin{aligned}
& \rho c \frac{\partial \vartheta}{\partial t}=\frac{1}{r} \frac{\partial}{\partial r}\left(r \lambda \frac{\partial \vartheta}{\partial r}\right)+\frac{\partial}{\partial z}\left(\lambda \frac{\partial \vartheta}{\partial z}\right) \\
& a_{P} \vartheta_{P}^{1}=a_{N} \vartheta_{N}^{1}+a_{S} \vartheta_{S}^{1}+a_{T} \vartheta_{T}^{1}+a_{B} \vartheta_{B}^{1}+a_{P}^{0} \vartheta_{P}^{0}
\end{aligned}
$$

\section{Results}

The results obtained by means of the numerical model, with thermographic measurement as the input parameters, show good accordance with the ones obtained by thermocouple measurement at referent points of the probe. Figures 7, 8 and 9 show this accordance during a cooling cycle in the air stream, velocity $3 \mathrm{~m} / \mathrm{s}$ for thermocouples numbered at positions 2,3 and 7 in fig. 3 . According to the shown accuracy it could be concluded that similar should be estimated for every single point inside the probe. Figure 10 shows temperature distribution in the cross section of the probe exposed to heating, after $1600 \mathrm{~s}$. The similar temperature distribution could be obtained as a result of every single numerical time step. That means that it is possible to draw cooling curves in CCT diagram for the each single point inside probe. Figure 11 shows calculated local heat transfer coefficient for the probe exposed to heating. On the basis of that data it is possible to find out the influence of heat transfer intensity on heating or cooling processes and it is also possible to simulate similar processes without performing measurements.

\section{Conclusion}

The solution of the problem in heat treatment of metals, gives a possibility to predict and follow temperature change for each single point inside the probe and allows the control of the system for heat treatment in a way which will guarantee that the desired microstructure is obtained. This gives a possibility to simulate similar processes without providing measurements, which reduces costs and time. Combination of numerical methods and infrared thermography gives a numerous advantages in comparison with classical methods.

\section{References}

[1] SUNDOV I., "Analysis of heat transfer and determination of non steady temperature fields inside complex geometry bodies using thermography", Thesys, Zagreb 1999

[2] SVAIC S. and, SUNDOV I. "IR Thermography and Heat Treatment of Metals", QIRT 94, Eurotherm No 42, Sorrento, Italy, 1994, p. 96-100.

[3] ANDERSON D.A. and TANNEHILL J.C. and PLETCHER, R.H. "Computational Fluid Mechanics and Heat Transfer", Hemisphere Publishing Co., Mc Grow-Hill Book Co. Inc., Washington, 1989.

[4] CLOIREC B. and JACQUOT P. and PLARD C. and BLAISE J.C. and SOUCHARD J. P. "Numerical modeling and optimization of metal parts Vacuum Heat treatment", Proceeding of International Heating Conference: Equipment and Processes, Schaumburg,99-103

[5] BASS R. and LEONARD D. and ALLEN M and BENNETT J.Jr.and CROSS M. and MORRAL J. and BROWN K., Heat transfer of turbine disks in a liquid quench (Part 1, 2, 3), $17^{\text {th }}$ ASM Heat treating Society Conference, Indiananpolis, 1997 
http://dx.doi.org/10.21611/qirt.2000.030

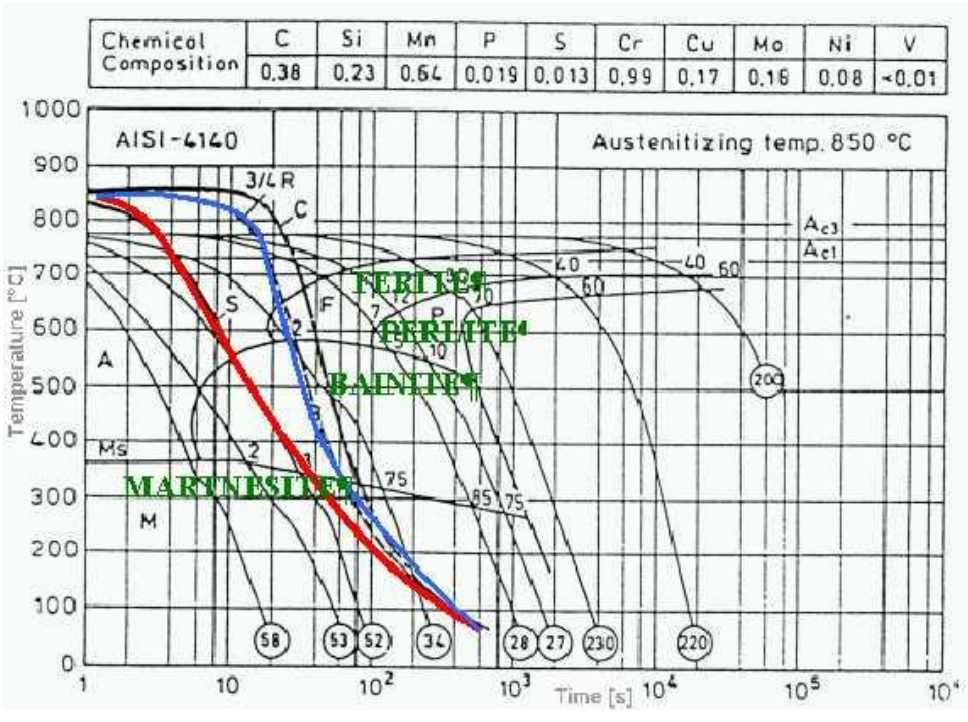

Figure 1 : CTT diagram

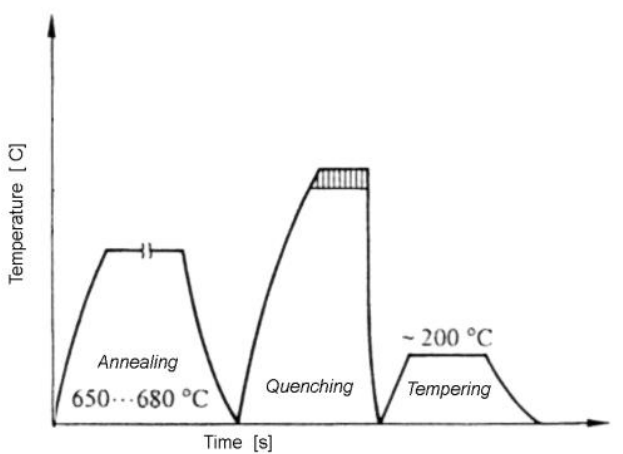

Figure 2: Complete heat treatment cycle

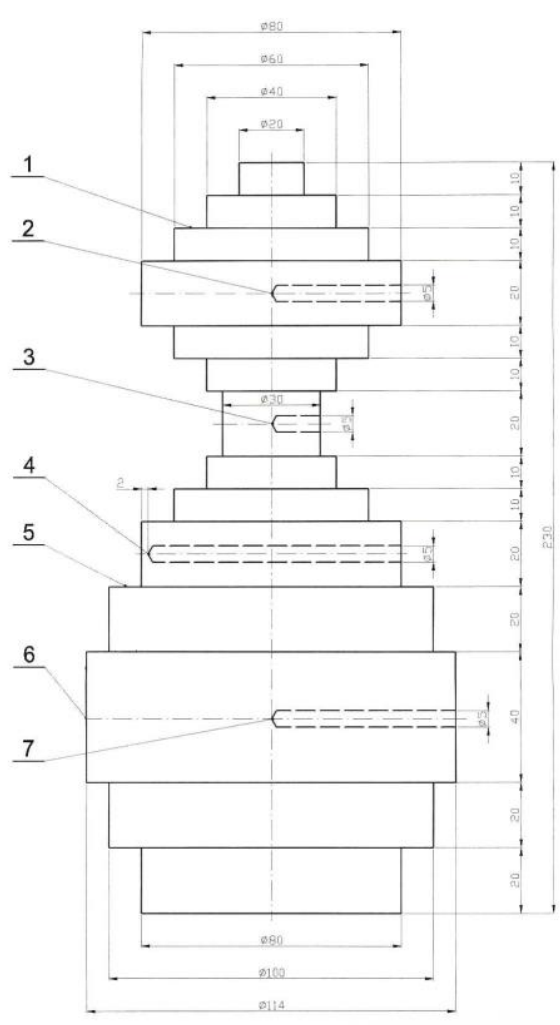

Figure 3: Dimensions of the probe and thermocouple positions

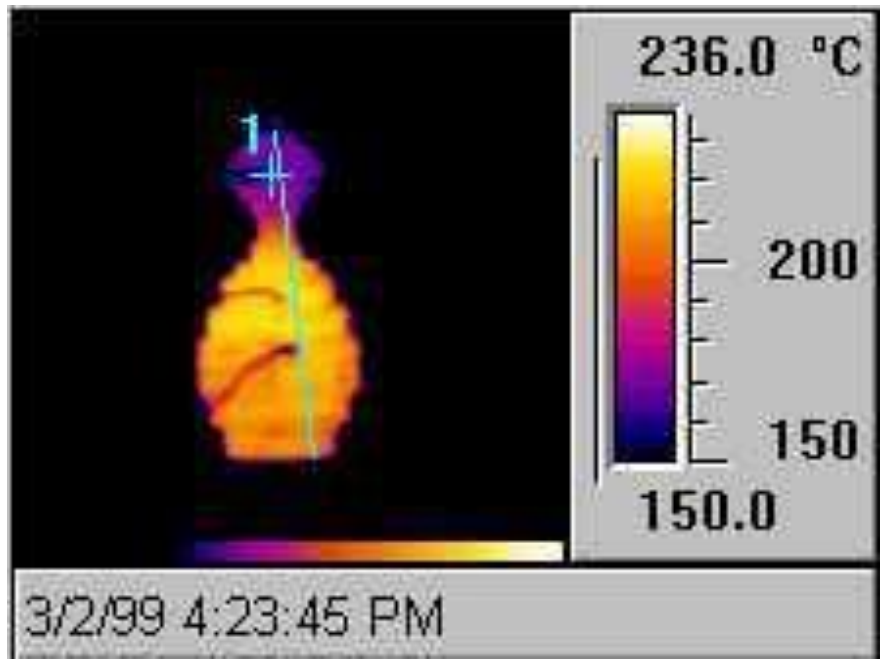

Figure 4. Thermogram of the probe during cooling in the air stream 


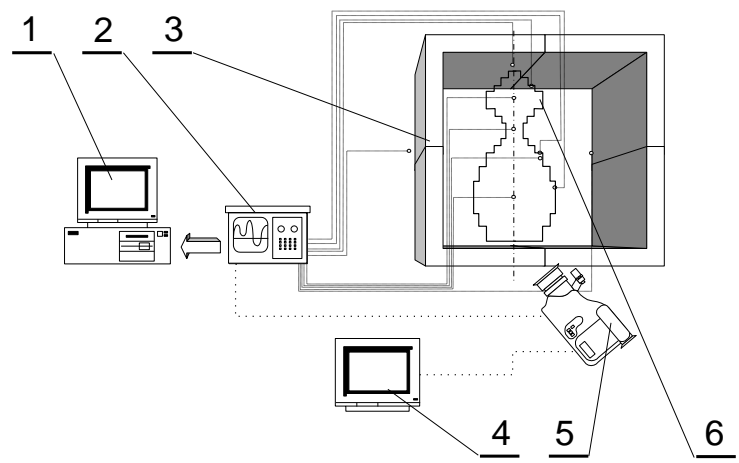

Figure 5: Experimental rig for heating

1) $P C$, 2) AD converter, 3) Furnace 4) Monitor, 5) IR camera, 6) Probe

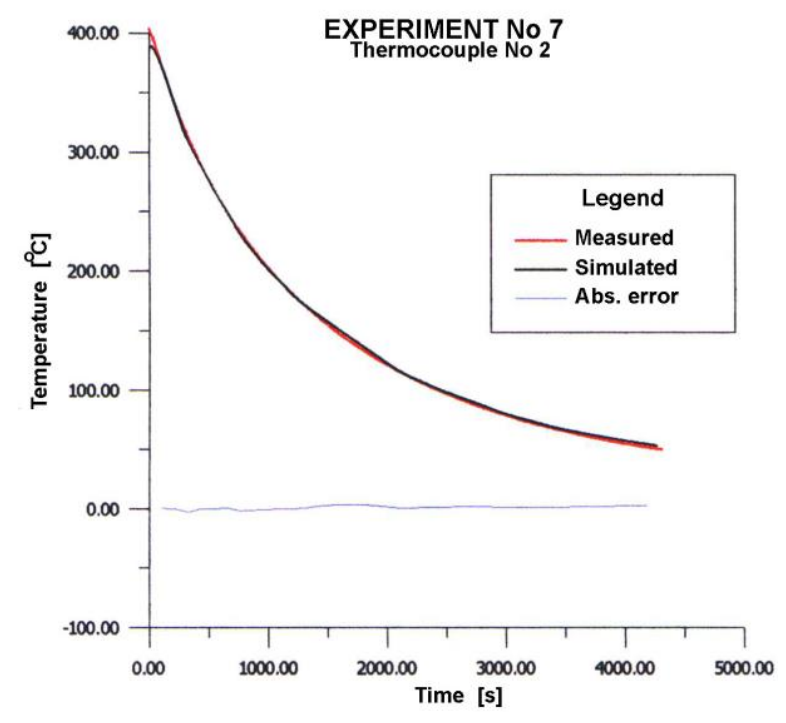

Figure 7: Measured and calculated temperatures

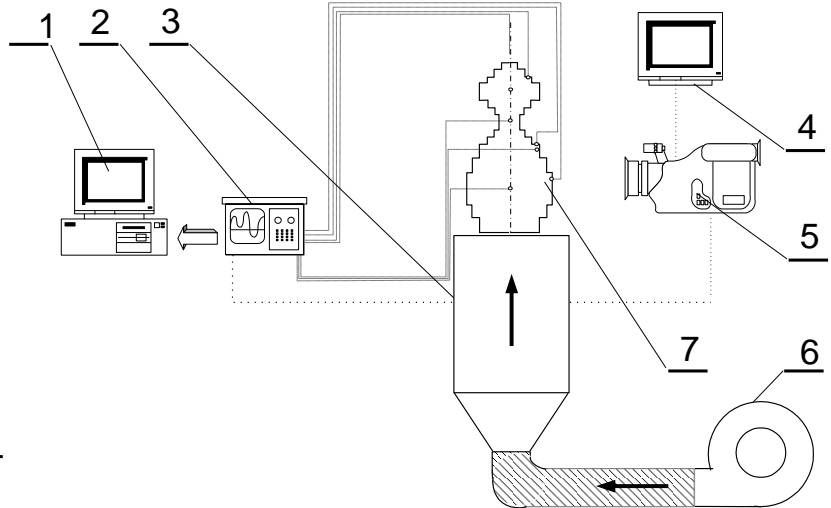

Figure 6. Experimental rig for cooling 1) PC, 2) AD converter, 3) Air duct 4) Monitor, 5) IR camera, 6) Fan, 7) Probe

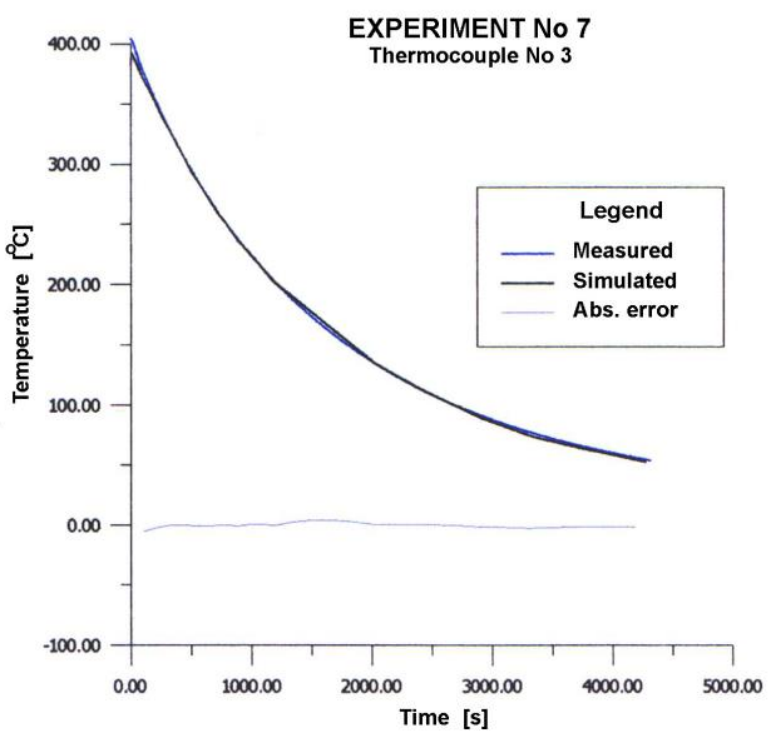

Figure 8. Measured and calculated temp.

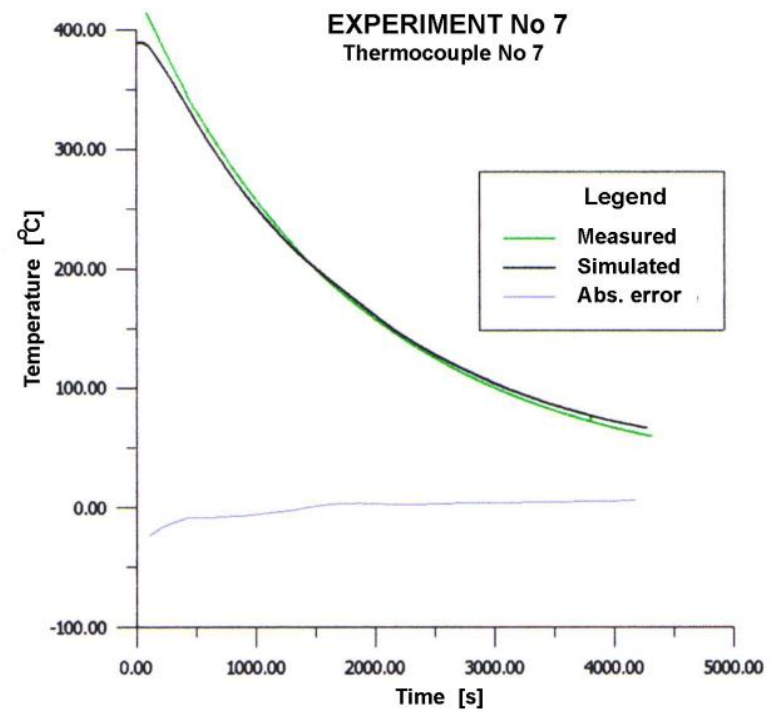

Figure 9: Measured and calculated temperatures 

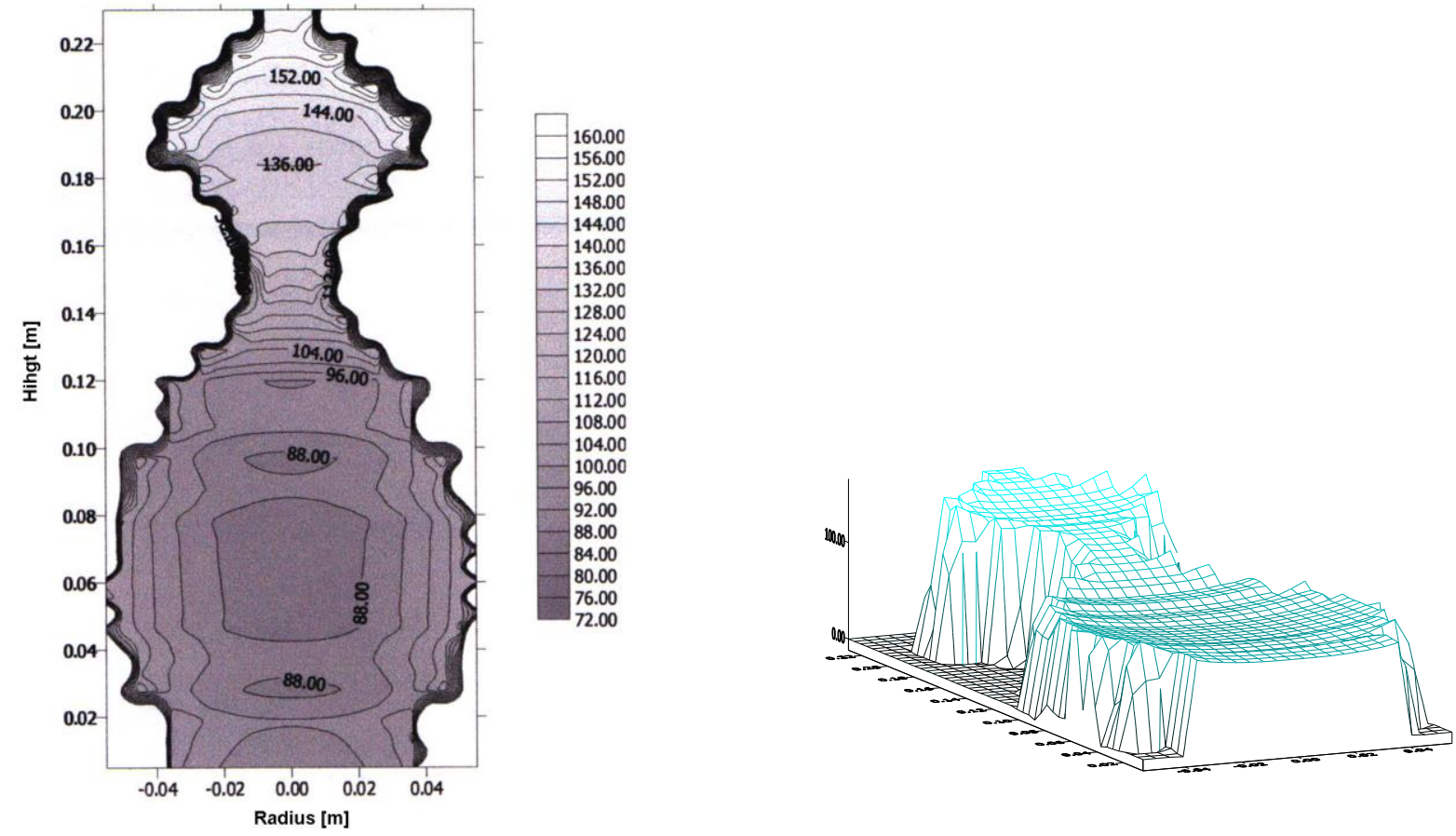

Figure 10: Temperature distribution in cross section of the probe after $1600 \mathrm{~s}$, heating

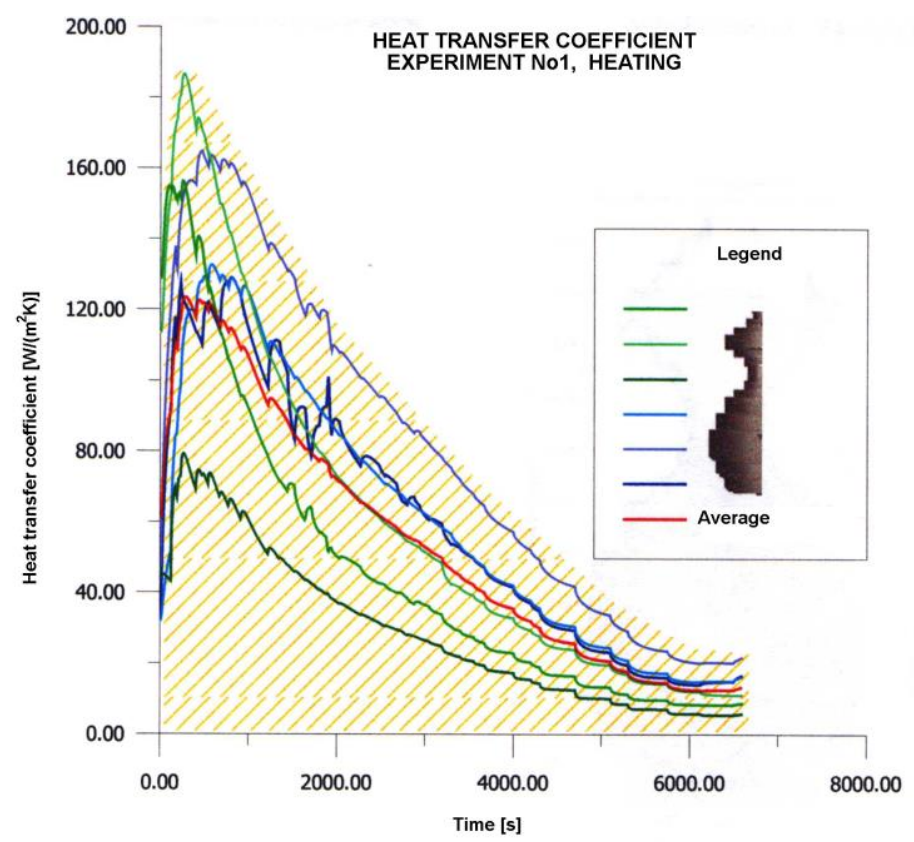

Figure 11: Local heat transfer coefficients, experiment No1, heating 